\title{
Quasi-black holes: general features and purely field configurations
}

\author{
K. A. Bronnikov* \\ VNIIMS, 46 Ozyornaya St., Moscow 119361, Russia, \\ Institute of Gravitation and Cosmology, PFUR, 6 Miklukho-Maklaya Street, Moscow 117198, Russia, and \\ National Research Nuclear University "MEPhI" (Moscow Engineering Physics Institute) \\ O. B. Zaslavskii ${ }^{\dagger}$ \\ Kharkov V.N. Karazin National University, 4 Svoboda Square, Kharkov 61022, Ukraine and \\ Institute of Mathematics and Mechanics, Kazan Federal University, 18 Kremlyovskaya St., Kazan 420008, Russia
}

\begin{abstract}
Objects that are on the threshold of forming the horizon but never collapse are called quasi-black holes (QBHs). We discuss the properties of the general spherically symmetric QBH metric without addressing its material source, including its limiting cases as the corresponding small parameter tends to zero. We then show that QBHs can exist among self-gravitating configurations of electromagnetic and dilatonic scalar fields without matter. These general results are illustrated by explicit examples of exact solutions.
\end{abstract}

PACS numbers: 04.70.Bw, 97.60.Lf

\section{INTRODUCTION}

During the recent two decades, the zoo of relativistic objects has increased and now, in addition to stars, black holes (BHs), wormholes, boson stars etc., it also includes the so-called quasi-black holes (QBHs). These are objects which are close to forming an event horizon but do not form it. Their more rigorous definition and general properties are described in [1]. There are different types of sources that provide physical realizations of QBHs. They naturally appear in the so-called Majumdar-Papapetrou (MP) systems [2, 3] made of extremely charged dust, where gravitational attraction is balanced by electric repulsion, $\rho_{e}= \pm \rho_{m} \rho_{m}$ and $\rho_{e}$ are the mass and charge densities, respectively (see [4] and references therein). Generalized MP systems including a dilaton scalar field (dilatonic MP, or DMP systems) have been suggested as QBH sources in [5, 6].

The goal of the present paper is twofold. First, we summarize and generalize the previous observations and describe the general properties of QBH metrics in the framework of general relativity, independently of their specific material source. Second, we show that QBHs can exist without (macroscopic) matter at all, as a result of combined action of gravitational, electromagnetic and scalar fields. It is worth noting that purely field QBHs have already been considered in $[7,8]$, being built with the aid of non-Abelian gauge and Higgs fields (selfgravitating monopoles). The main results of these papers were based on numerical calculations, so it is not easy to control them. A similar behavior was found analytically in [9] using thin shells made of extremal dust.

Unlike that, we will deal with Abelian fields only, which enables us to trace the properties of QBHs analytically

\footnotetext{
*Electronic address: kb20@yandex.ru
}

$\dagger$ Electronic address: zaslav@ukr.net and in more detail. To the best of our knowledge, this is a first example where a QBH is (i) purely field (vacuum), (ii) built using Abelian fields.

In Section II we will discuss the properties of the general QBH metric without addressing its material source. Section III is devoted to purely field QBH models, and Section IV is a conclusion. Throughout the paper, we use the units in which $G=c=1$.

\section{GENERAL FEATURES OF QBH METRICS}

In both MP and DMP systems, in the case of spherical symmetry the metric has the form $[2,3,5,6]$

$$
d s^{2}=A(x) d t^{2}-A(x)^{-1}\left(d x^{2}+x^{2} d \Omega^{2}\right),
$$

where $d \Omega^{2}=d \theta^{2}+\sin ^{2} \theta d \varphi^{2}$ is the linear element on a unit sphere.

It turns out that this particular form of the metric (1) entails some important consequences which are insensitive to the details of a material source that supports the metric. To discuss them, in this section we will "forget" physical motivations for the metric (1) and look at it from a purely geometric viewpoint.

To begin with, the metric (1) describes a $\mathrm{BH}$ if $A(x)$ has a regular zero at some value of $x, x=x_{\text {hor }}$, corresponding to the event horizon. A simple inspection shows that this can only be achieved at finite values of the spherical radius $r(x)=x / \sqrt{A(x)}$ if and only if

$$
x_{\text {hor }}=0, \quad A(x)=x^{2} A_{1}(x),
$$

with a smooth function $A_{1}(x)$ such that $A_{1}(0) \neq 0$.

Further, the system admits the existence of QBHs: to be close to a $\mathrm{BH}$, such a configuration should contain a region with small $A(x)$ but have a regular center. Introducing a small parameter $c$, we can describe the situation by presenting $A(x) \mapsto A(x, c)$ as a Taylor series

$$
A(x, c)=A_{0}(c)+\frac{1}{2} A_{2}(c) x^{2}+\ldots,
$$


where $A_{0}(c) \rightarrow 0$ as $c \rightarrow 0$ while $A_{2}(0)$ is finite. Redefining the parameter $c$ so that $A_{0}(c)=\frac{1}{2} A_{2}(0) c^{2}$, we can put without loss of generality

$$
A(x, c)=\frac{x^{2}+c^{2}}{f^{2}(x, c)} \quad \Rightarrow \quad r(x, c)=\frac{x f(x, c)}{\sqrt{x^{2}+c^{2}}},
$$

where $f(x, c)$ is a smooth function with a well-defined nonzero limit as $c \rightarrow 0$.

This reasoning is the same as was applied to charged dust configurations in $[1,4]$. Let us stress that in the general case, with the metric function (4), the region where the "redshift function" $A(x, c)$ is small, is itself not small at all: assuming that $f(x, c)=O(1)$ and $c \ll 1$, it is easily shown that the radius $r(c)$ of the sphere $x=c$ is $f(c, c) / \sqrt{2}=O(1)$; the distance from the center to this sphere and the volume of the ball $x \leq c$ are also $O(1)$.

\section{A. Scales and regions in $\mathrm{QBH}$ spacetimes}

We will assume that all metrics are asymptotically flat, so that $A \rightarrow 1$ as $x \rightarrow \infty$, although the QBH concept is meanginful even without this assumption.

According to [1] and [10], one of the typical features of QBHs consists in that there are at least two regions with their own characteristic scales on which physical and geometric quantities like density, metric coefficients, etc. may vary. If there is a sharp boundary between, say, matter and vacuum as in Bonnor stars [11, 12], the difference between these two scales becomes as large as one likes when the boundary approaches the position of a wouldbe horizon. For spherically symmetric spacetimes it is demonstrated explicitly in Sec. III of [1]. In a more general setting, without spherical symmetry, this was traced in [10], where the terms "inner world" and "outer world" were used. Meanwhile, even if there is no sharp boundary between two regions of space-time, two scales and associated regions appear anyway. Moreover, it also makes sense to single out an intermediate region corresponding to the immediate vicinity of a would-be horizon. Instead of "inner and outer worlds", in our view, it is better (though also not quite precise) to speak of looking at a certain region "through a microscope". This expression will be explained below.

Now, we will generalize the observations concerning the QBH metrics, previously made for different specific material sources (dust, electromagnetic and scalar fields, see $[5,6]$. Actually, they follow directly from the form (1) of the metric. It appears that there is a natural division of the whole space into three regions.

\section{B. Region 1 (central), $0 \leq x \lesssim c$}

Let us try to preserve a regular center in the limit $c \rightarrow 0$. To do so, we introduce the new coordinates

$$
X=x / c, \quad T=c t / f_{0}, \quad f_{0}:=f(0,0),
$$

and, rewriting the metric (1) with (4) in terms of these new coordinates, consider $c \rightarrow 0$. The result is

$$
d s^{2}=\left(1+X^{2}\right) d T^{2}-\frac{f_{0}^{2}}{1+X^{2}}\left(d X^{2}+X^{2} d \Omega^{2}\right) .
$$

This limiting metric is geodesically complete, it still has a regular center at $X=0$, but it is not asymptotically flat: instead, at large $X$ it approaches a flux-tube metric with $r(X)=$ const and $g_{T T} \rightarrow \infty$, which can be characterized as a repulsive infinity for test particles.

Even though the transformation (5) applies to the whole space, the metric (6) can be seen as a result of infinitely stretching the neighborhood of the center (which explains the above mentioning of a "microscope"). Noteworthy, it does not depend on the particular form of the function $f(x, c)$ and even on $f(x, 0)$ : the only remaining parameter is the constant $f_{0}$.

\section{Region 2 (intermediate, close to a quasihorizon)}

Consider the values of $x$ such that the spherical radius $r(x, c)$ in (4) corresponds to the horizon size of a BH obtained in a direct limit $c \rightarrow 0$. It makes sense to call some properly defined sphere from this region a "quasihorizon". There can be at least two reasonable definitions of a quasihorizon in terms of $c$ and the function $f(x, c)$ from the ansatz (4):

First, a quasihorizon $x=x_{1}$ can be defined as such a value of $x$ that the radius $r\left(x_{1}, c\right)$ is simply equal to the $\mathrm{BH}$ horizon radius $r_{h}$. In the limit $c \rightarrow 0$ in (4), we have $r(x)=f(x, 0)$, and the horizon is located at $x=0$, therefore $r_{h}=f(0,0)=f_{0}$, and $x_{1}$ should satisfy the equation $r\left(x_{1}, c\right)=f_{0}$.

Second, a quasihorizon can be defined using the fact that a horizon is a surface where $V \equiv-g^{a b} r_{, a} r_{, b}=0$ (that is, the gradient of the function $r(x, t)$ considered as a scalar field in the 2D space parametrized by $x$ and $t$ becomes null). In our static case we have $V=A(d r / d x)^{2}$, accordingly a horizon corresponds to $A=0$. In a $\mathrm{QBH}$ metric, $V$ nowhere turns to zero but has a small minimum. to be called a quasihorizon ${ }^{1} x=x_{2}$.

Let us find $x_{1}$ and $x_{2}$ using the representation (4). We assume that they are small in terms of $c$ (since the very existence of these surfaces is connected with $c \neq 0$ ) but still $x_{1,2} \gg c$ (an assumption to be verified by the results).

Moreover, we will use the first two terms of the Taylor expansion of $f(x, c)$ near $x=0$, neglecting its $c$ depen-

\footnotetext{
${ }^{1}$ If one uses $r$ as the radial coordinate (the usual Schwarzschildlike coordinate), then $-V$ coincides with the metric coefficient $g^{r r}$. The notion of a quasihorizon as a minimum of $\left|g^{r r}\right|$ was used in $[1,4]$.
} 
dence due to $c \ll x$. Thus we have ${ }^{2}$

$$
f(x, c) \approx f(x)=f_{0}+f_{1} x+\ldots
$$

where $f_{0}, f_{1}, \ldots$ are constants. form

Now, the equation $r\left(x_{1}, c\right)=f_{0}$ is rewritten in the

$$
\frac{x_{1}}{\sqrt{x_{1}^{2}+c^{2}}}\left(f_{0}+f_{1} x_{1}\right)=1,
$$

from which, for small $c$, it follows

$$
x_{1}=a_{1} c^{2 / 3}, \quad a_{1}=\left(2 f_{1} / f_{0}\right)^{-1 / 3} .
$$

To find $x_{2}$, we note that according to (1) and (4) (the prime denotes $d / d x)$,

$$
V=A r^{\prime 2}=\left(1-x \gamma^{\prime}\right)^{2}, \quad 2 \gamma \equiv \ln A
$$

therefore the condition $V^{\prime}=0$, necessary for a minimum of $V$, reads

$$
\left(x \gamma^{\prime}\right)^{\prime}=0, \quad \text { or } \quad x \gamma^{\prime \prime}=-\gamma^{\prime} .
$$

Substituting $A \equiv e^{2 \gamma}$ according to (4) and the expansion (7) for $f(x)$, we arrive at the equation $f_{1} / f_{0}=2 c^{2} / x_{2}^{3}$, whence

$$
x_{2}=a_{2} c^{2 / 3}, \quad a_{2}=\left[f_{1} /\left(2 f_{0}\right)\right]^{-1 / 3} .
$$

Moreover, it can be verified that $V^{\prime \prime}>0$ at $x=x_{2}$, which confirms that it is a minimum, and that possible inclusion of higher terms in the Taylor expansion (7) does not break these results.

Thus $x_{1}$ and $x_{2}$ coincide by order of magnitude, the difference being only in the factors $a_{1,2}$. Generically $a_{1,2}=O(1)$, hence $x_{1,2}=O\left(c^{2 / 3}\right) \gg c$, as was assumed.

The $c$ dependence of $x_{1,2}$ prompts a possible transformation that could help us to approximately preserve the geometry near $x=x_{1,2}$ in the limit $c \rightarrow 0$ : let us substitute instead of $x$ and $t$ the coordinates

$$
\xi=c^{-2 / 3} x, \quad \tau=f_{0}^{-1} c^{2 / 3} t,
$$

and consider the limit $c \rightarrow 0$ in these new coordinates. The result is

$$
d s^{2}=\xi^{2} d \tau^{2}-\frac{f_{0}^{2}}{\xi^{2}} d \xi^{2}-f_{0}^{2} d \Omega^{2} .
$$

It is a pure flux-tube metric, coinciding up to a constant factor with the Bertotti-Robinson metric $[15,16]$ in agreement with Eq. (30) of [1]. Its peculiar feature is that, although $r$ is constant, the metric is strongly $\xi$-dependent. Instead of a regular center, it contains a

\footnotetext{
${ }^{2}$ It is the generic case, but similar results are obtained if the second nonvanishing term in (7) has the form $f_{n} x^{n}, n \in \mathbb{N}$. In particular, the limiting metric for $c \rightarrow 0$ still has the form (13).
}

second-order horizon at $\xi=0$, and the region $\xi<0$ beyond it is an exact copy of the region $\xi>0$. Recall that the Bertotti-Robinson metric approximately describes the throat-like neighborhood of the extremal ReissnerNordström (RN) metric, but, at the same time, it is an exact solution to the Einstein-Maxwell equations and can be considered without reference to the $\mathrm{RN}$ metric.

The metric (13) at $x>0$ can be called a "microscope image" of the quisihorizon domain of a $\mathrm{QBH}$ in the following sense. While $r=$ const in the limiting metric, it changes comparatively slowly in the QBH metric near $x=x_{1}$ or $x=x_{2}$ : indeed, at $x=a c^{2 / 3}$, where $a=O(1)$, one has $r^{\prime} / r=O(1)$ while $\gamma^{\prime}=A^{\prime} /(2 A)=O\left(c^{-2 / 3}\right)$, a large quantity. That is, $r(x)$ is changing in this region much slower than $A(x)$.

\section{Region 3, $x \gtrsim x_{1,2} \gg c$.}

In this outer region, one can simply put $c=0$ without changing the qualitative features of the metric, so it looks like that of a $\mathrm{BH}$.

Thus we obtain different limiting metrics preserving the main properties of different regions of a QBH spacetime. For instance, there is a limiting metric preserving a regular center but having no spatial infinity, and some authors even describe such a situation saying that near the center "there exists another world on its own right, with another metric" [10].

Summarizing, we can say that although each kind of limiting procedure touches upon the whole QBH spacetime, different limits preserve the geometric properties of different parts of space. Thus, the straightforward limit $c \rightarrow 0$ well preserves the geometry at large radii, including asymptotic flatness, while the other two transitions correspond to "looking through a microscope" at the central (1) and intermediate (2) regions. It is of interest that these both limiting metrics are universal, insensitive to the detailed behavior of the function $f(x, c)$. At small but nonzero $c$ these three regions overlap and cover the whole space. The limiting metrics can also be considered on their own rights as solutions to the field equations.

\section{E. QBHs with boundaries}

So far we assumed that there is a unique metric valid in the whole space. This assumption can correspond either to purely field systems (to be exemplified in Section 3) or to matter distributions smoothly vanishing at large distances from the center.

Meanwhile, many of the existing QBH models represent starlike objects, such as, e.g., Bonnor stars [11] that are compact objects made of extremely charged dust surrounded by vacuum. We can also consider such objects in a general form. Then the metrics are different in the inner and outer regions and are mutually independent up to the necessity to match them at the junction surface. 
For the whole such configuration to be close to a $\mathrm{BH}$ one, it is sufficient to require the behavior (4) of the metric functions in the outer region only (in fact, the outer metric can be a pure $\mathrm{BH}$ one, as happens, e.g., in models using the extreme RN metric for this purpose [11]). However, in a $\mathrm{QBH}$ the junction surface should be close to a would-be horizon, and this value of $x$ may be chosen as the small parameter of the system: the inner region is then $0 \leq x \leq c$, where $x=0$ is the center. It then follows that at the junction the external function $g_{00}=A_{\text {ext }}(x, c)$ should take a value $A_{\text {ext }}(c, c)=O\left(c^{2}\right)$.

The function $g_{00}=A(x, c)$ in the central region should satisfy the following conditions: (i) a regular center, hence $A(x, c) \approx A_{0}(c)+A_{2}(c) x^{2}$ as $x \rightarrow 0$, and (ii) a smooth junction, hence $A(c, c)=A_{\text {ext }}(c, c)$ and $A^{\prime}(c, c)=$ $A_{\text {ext }}^{\prime}(c, c)$ (as before, the prime means $d / d x$ ). In other respects, $A(x, c)$ is arbitrary.

Consider the outer metric function $A(x, c)(x \geq c)$ in the RN form, so that

$$
A_{\mathrm{ext}}=\frac{x^{2}}{(x+m)^{2}}
$$

Inside, at $0 \leq x \leq c$, we can choose $A(x, c)$ in the form

$$
A=\frac{c^{2}}{[c+m+m \eta(y)]^{2}}, \quad y=x / c .
$$

The two functions (hence the metric itself) are smoothly matched at $x=c$ provided that

$$
\eta(1)=0, \quad \frac{d \eta}{d y}(1)=-1
$$

while a regular center at $y=0$ requires $\eta=\eta_{0}+o(y)$ at small $y$. This construction generalizes Bonnor's example [11] in which

$$
\eta(y)=\left(1-y^{n}\right) / n, \quad n \geq 2 .
$$

It is of course quite easy to invent a diversity of such metrics. One more good example is

$$
\eta=1-e^{\left(y^{2}-1\right) / 2}
$$

After the substitutions $x=y c, t=T / c$, in the limit $c \rightarrow 0$, the metric in region 1 acquires the form

$$
d s^{2}=\frac{d T^{2}}{m^{2}(1+\eta)^{2}}-m^{2}(1+\eta)^{2}\left(d y^{2}+y^{2} d \Omega^{2}\right)
$$

so that the general form (1) is preserved. Thus the limiting space-times of QBHs with boundaries are not unique and even contain an arbitrary function $\eta(y)$.

\section{QBHS WITH SCALAR AND ELECTROMAGNETIC FIELDS}

So far we have been only discussing the metric properties of QBHs connected with the ansatz (1). In what follows we will consider an example of a purely field system that can realize a full description of such static, spherically symmetricconfigurations. The Lagrangian is taken in the form

$$
L=\frac{1}{16 \pi}\left[R+2 \varepsilon(\partial \chi)^{2}-F^{2} P(\chi)\right],
$$

where $\varepsilon=1$ for a normal scalar field, $\varepsilon=-1$ for a phantom one, $\varepsilon= \pm 1, F^{2} \equiv F^{\alpha \beta} F_{\alpha \beta}$ with $F_{\mu \nu}=\partial_{\mu} A_{\nu}-\partial_{\nu} A_{\mu}$ the electromagnetic field tensor, $A_{\mu}$ the vector potential, $\chi$ a dilatonic scalar field. For generality, and to provide correspondence with $[13,14]$, we do not fix the sign of $P(\chi)$. The metric is assumed in the form (1) and contains only one unknown function ${ }^{3} A(x) \equiv e^{2 \gamma(x)}$. The electromagnetic field is chosen in the most general form compatible with spherical symmetry (only radial electric and magnetic fields, characterized by the charges, $q_{e}$ and $q_{m}$ are possible), the Maxwell-like equation leads to the squared electric field strength $F^{01} F_{10}=q_{e}^{2} /\left[r^{4} P^{2}(\chi)\right]$, and the remaining field equations may be written in the form

$$
\begin{aligned}
x^{4} \gamma^{\prime \prime}+2 x^{3} \gamma^{\prime} & =N e^{2 \gamma}, \\
2 \varepsilon\left(x^{4} \chi^{\prime \prime}+2 x^{3} \chi^{\prime}\right) & =e^{2 \gamma} \frac{d N}{d \chi}, \\
x^{4}\left(\gamma^{\prime 2}+\varepsilon \chi^{\prime 2}\right) & =N(\chi) e^{2 \gamma}
\end{aligned}
$$

where $N \equiv q_{e}^{2} / P(\chi)+q_{m}^{2} P(\chi)$.

\section{A. Field configurations with a regular center}

We are interested in asymptotically flat solitonic, or particlelike configurations with a regular center. If, in addition, $g_{t t} \ll 1$ in some region of space (the gravitational redshift of emitted signals reaches large values at infinity where $g_{t t}=1$ ), we have a $\mathrm{QBH}$.

It is in general hard to solve Eqs. (21)-(23) with nontrivial $P(\chi)$ even if one of the charges, electric or magnetic, is zero. However, one can obtain the general result that if there is a regular center, it corresponds to $x=0$ and $N \sim x^{4}$ near it.

Indeed, it can be shown that a regular center in this system can exist if the metric has the form (1), and this center evidently corresponds to $x=0$ where $e^{2 \gamma} \equiv$ $A(x)=A_{0}+\frac{1}{2} A_{2} x^{2}+o\left(x^{2}\right)$, where $A_{0}>0$. Substituting it into (21) and (23), we obtain at small $x$

$$
N(\chi)=\frac{3 x^{4} A_{2}}{2 A_{0}^{2}}+o\left(x^{4}\right), \quad \varepsilon \chi^{\prime 2}=\frac{3 A_{2}}{2 A_{0}}+o(1) .
$$

Thus we have proved that $N=0$ at the center and, in addition, that in the generic case $A_{2} \neq 0$ we have

\footnotetext{
${ }^{3}$ More general static systems without spatial symmetry and an arbitrary dependence $\gamma\left(x^{i}\right)(i=1,2,3)$ in the presence of scalarly and electrically charged dust have been considered in $[5,6]$, with an electric potential $\phi\left(x^{i}\right)$ and a dilatonic field $\chi\left(x^{i}\right)$.
} 
$\varepsilon=\operatorname{sign} A_{2}$. So, with a normal (non-phantom) scalar field $(\varepsilon=+1)$ there is a minimum of $A(x)$ at the center.

Moreover, the expression for $N(\chi)$ in terms of $P(\chi)$ shows that it is impossible to obtain $N=0$ if both $q_{e} \neq 0$ and $q_{m} \neq 0$. We thus have the following imortant result: Configurations with a regular center are possible with an

electric charge (then $P(\chi)=\infty$ at the center) or with a magnetic charge (then $P(\chi)=0$ at the center) and are impossible if both charges are nonzero.

Thus, using $N(\chi)$ in the description of QBHs, we have only two options: either $N(\chi)=q_{e}^{2} / P(\chi)$ or $N(\chi)=$ $q_{m}^{2} P(\chi)$.

It is of interest to compare the Maxwell-dilaton fields and nonlinear electrodynamics (NED) with the Lagrangian $L(F), \quad F:=F_{\mu \nu} F^{\mu \nu}$ as sources of gravity in what concerns the existence of self-gravitating configurations with a regular center $[17,18]$. In both cases solutions with both electric and magnetic charges and a regular center are impossible (unless the NED Lagrangians are different in different parts of space), but with NED the same is true for a purely electric source; on the other hand, in both theories, a regular center requires a singular behavior of the interaction Lagrangian: thus, in purely magnetic solutions with NED a regular center requires $L(F) \rightarrow \infty$, whereas in EMD one needs $P \rightarrow 0$.

\section{B. Examples}

Example 1. Purely field configurations do not contain boundaries (unless there are phase transitions), so we return to the general metric (1) under the condition (4). Let us choose

$$
e^{2 \gamma} \equiv A(x)=\frac{x^{2}+c^{2}}{\left(m+\sqrt{x^{2}+c^{2}}\right)^{2}},
$$

where $m>0$ and $c>0$ are constants. Such a trial function was used in [1,4], but the material source was different there (extremal dust). Then at large $x$ we have $A \approx 1-2 m / x+3 m^{2} / x^{2}+\ldots$, hence $m$ has the meaning of a Schwarzschild mass. Near the center

$$
A(x)=\frac{c^{2}}{(m+c)^{2}}+\frac{m x^{2}}{(m+c)^{3}}+\ldots,
$$

which confirms that the center is regular and also that with $m>0$ the solution corresponds to a normal scalar field. Meanwhile, the direct limit $c \rightarrow 0$ leads to $A(x)=$ $x^{2} /(x+m)^{2}$, that is, the extreme RN metric with a double horizon at $x=0, P \equiv 1$ and $\chi^{\prime} \equiv 0$.

From (21)-(23) we obtain the following expressions for $N(\chi)$ and $\chi^{\prime}$ :

$$
\begin{aligned}
N(\chi) & =\frac{m x^{4}}{z^{6}}\left[m x^{2}+3 c^{2}(m+z)\right], \\
\chi^{\prime 2} & =\frac{3 c^{2} m}{z^{4}(m+z)},
\end{aligned}
$$

where $z=\sqrt{x^{2}+c^{2}}$. The function $\chi(x)$ is then obtained in terms of elliptic functions, and the dependence $N(\chi)$ cannot be found explicitly.

As follows from the above-said, the solution contains either an electric or a magnetic charge and cannot contain both. In a purely electric solution, we have $N(\chi)=$ $q_{e}^{2} / P(\chi)$, hence $P \rightarrow \infty$ at the regular center. On the contrary, in a purely magnetic solution, we have $N(\chi)=q_{m}^{2} P(\chi)$, and $P(0)=0$.

The limiting transitions $c \rightarrow 0$ for this example of a QBH gives in region 1 the metric (6) with $f_{0}=m$. As to region 2 , at $x \gg c$ we have $f(x, c) \approx m+x$, so that in the scheme of Sec. IIC we have $f_{0}=m, f_{1}=1$. Accordingly, for the quasihorizon (by the two definitions in Sec. IIC) we obtain $x_{1}=(m / 2)^{1 / 3} c^{2 / 3}$ and $x_{2}=(2 m)^{1 / 3} c^{2 / 3}$, and the substitution (12) again leads to the limiting metric (13).

Example 2. Consider the exact solitonic (particlelike) solution to the present field equations found in [19], see also [18]. We reproduce it here (in slightly changed notations) and discuss it from the viewpoint of QBHs, assuming for definiteness that there is only an electric charge and consequently $N(\chi)=q^{2} / P(\chi)$.

The solution is characterized by the following relations:

$$
\begin{aligned}
A(x) \equiv e^{2 \gamma(x)} & =\frac{\left(1-\delta^{2}\right)^{2}}{\left(1-\delta^{2} e^{-b / x}\right)^{2}}, \\
\chi(x) & =\chi_{0}-2 \arcsin \left[\delta e^{-b /(2 x)}\right], \\
P(\chi) & =\frac{\sin ^{2}\left(\chi_{0} / 2\right)}{\sin ^{2}\left[\left(\chi-\chi_{0}\right)\right]},
\end{aligned}
$$

where $\sin \left(\chi_{0} / 2\right)=\delta=m /|q|, b=\left[q^{2}\left(1-\delta^{2}\right)\right] / m$.

This solution is asymptotically flat as $x \rightarrow \infty$ and has a regular center at $x=0$. All its parameters are expressed in terms of the mass $m$ and the electric charge $q$, and $m<|q|$, so that $\delta<1$, while the scalar $\chi$ changes from zero at infinity to $\chi_{0}$ at the center. The metric function $A(x)=e^{2 \gamma}$ changes from $\left(1-\delta^{2}\right)^{2}$ at the center to 1 at infinity.

Now, let us introduce the parameter $c$ according to $1-\delta^{2}=c / m$. The solution describes a QBH type configuration if $c / m \ll 1$. However, the behavior of $A(x)$ does not conform to the generic relations (3) and (4) since the function $e^{b / x}$ tends to zero as $x \rightarrow 0$ not in a power manner. Nevertheless, this system possesses the main properties of QBHs, that the size of a high-redshift region is not small, being of the same order of magnitude as the Schwarzschild radius $r_{s}=2 \mathrm{~m}$. Indeed, if we let $c \ll m$, the sphere $x=c$ belongs to the highredshift region since $\left.e^{\gamma}\right|_{x=c}=(c / m) e /(e-1)$; its radius is $\left.r\right|_{x=c}=m(e-1) / e \approx 0.632 m$; the distance of this sphere from the center is $\ell(0, c) \approx 0.851 \mathrm{~m}$.

In the direct limit $c \rightarrow 0$, assuming $x \gg c$ (which corresponds to region 3 ), the metric tends to that of the extreme RN BH $\left(e^{\gamma} \rightarrow x /(x+m)\right.$. Quite naturally, the field equations imply that in this limit the dilaton field becomes constant: $\chi=\chi_{0}-\pi$. 
To describe region 1, we introduce the new coordinates $z=m x / c$ and $\tau=c t / m$, and then in the limit $c \rightarrow 0$ the metric and the dilaton field become

$$
\begin{aligned}
d s^{2} & =\frac{d \tau^{2}}{\left(1-e^{-m / z}\right)^{2}}-\left(1-e^{-m / z}\right)^{2}\left(d z^{2}+z^{2} d \Omega^{2}\right),(31) \\
\chi & =\chi_{0}-2 \arcsin e^{-m /(2 z)}
\end{aligned}
$$

This metric behaves like (6): it has a regular center at $z=0$ and approaches a flux-tube form at large $z$.

As to a possible quasihorizon, it turns out that by both definitions 1 and 2 (see Section IIC) it now corresponds to $x \sim c$, hence it belongs to region 1 . Therefore in this case there are only two distinct regions 1 and 3 .

\section{CONCLUSION}

We have traced the general features of QBH spacetimes independent of their specific material sources, thus generalizing a number of previous observations. It turns out that one can distinguish there three typical regions: the immediate vicinity of a regular centre, the quasihorizon region, and the far asymptotically flat region. Different forms of limiting transition $c \rightarrow 0$ convert these regions to different space-times, each of them being, in general, geodesically complete. Mathematically, this can be viewed as examples of "limits of space-times" where the result of a limiting transition depends on how the parametersare entangled with coordinates [20].

An important particular class of QBHs is formed by purely field configurations. We have shown that, in addition to the MP or DMP systems, there is an alternative way of QBH construction. To the best of our knowledge, it is for the first time that QBHs are obtained due to the dilaton and electromagnetic fields only, without matter. These are purely field configurations which do not collapse even on the very threshold of forming a horizon but do not form it. This a somewhat unexpected result since it cannot be given a simple interpretation in the spirit of MP systems (a balance between gravitational attraction and electromagnetic repulsion). It has turned out, in particular, that one of the previously found exact solutions for coupled gravitational, scalar and electromagnetic fields with a regular center contains a $\mathrm{QBH}$ at proper values of the parameters.

It would be of interest to generalize the above results to rotating space-times.

\section{Acknowledgments}

The work by O.Z. was supported by the subsidy allocated to Kazan Federal University for the state assignment in the sphere of scientific activities.
[1] José P. S. Lemos and O. B. Zaslavskii, Phys. Rev. D 76, 084030 (2007).

[2] S.D. Majumdar, Phys. Rev. 72, 390 (1947).

[3] A. Papapetrou, Proc. R. Ir. Acad., Sect. A 51, 191 (1947).

[4] José P. S. Lemos and E. Weinberg, Phys. Rev. D 69, 104004 (2004).

[5] K.A. Bronnikov, J.C. Fabris, R. Silveira, and O.B. Zaslavskii, Phys. Rev. D 89, 107501 (2014).

[6] K.A. Bronnikov, J.C. Fabris, R. Silveira, and O.B. Zaslavskii, Gen. Rel. Grav. 46, 1775 (2014).

[7] A. Lue and E.J. Weinberg, Phys. Rev. D 60, 084025 (1999).

[8] A. Lue and E. Weinberg, Phys. Rev. D 61, 124003 (2000).

[9] José P.S. Lemos and V.T. Zanchin, J. Math. Phys. 47, 042504 (2006).

[10] R. Meinel and M. Hütten, Class. Quant. Grav. 28, 225010 (2011).
[11] W.B. Bonnor, Class. Quantum Grav. 16, 4125 (1999).

[12] W.B. Bonnor, Gen. Rel. Grav. 42, 1825 (2010).

[13] G. Clément, J.C. Fabris, and M.E. Rodrigues, Phys. Rev. D 79, 064021 (2009).

[14] M. Azreg-Ainou, G. Clément, J.C. Fabris, and M.E. Rodrigues, Phys. Rev 83, 124001 (2011); arXiv: 1102.4093.

[15] B. Bertotti, Phys. Rev. 116, 1331 (1959).

[16] I. Robinson, Bull. Acad. Pol. Sci. 7, 351 (1959).

[17] K.A. Bronnikov, Phys. Rev. D 63, 044005 (2001); grqc/0006014.

[18] K.A. Bronnikov, V.N. Melnikov, G.N. Shikin, and K.P. Staniukovich, Ann. Phys. (NY) 118 (1), 84 (1979).

[19] K.A. Bronnikov, V.N. Melnikov, and G.N. Shikin, Izvestiya Vuzov SSSR, Fiz., No. 11, 69 (1978); Russ. Phys. J. 21 (11), 1443 (1978).

[20] R. Geroch, Commun. Math. Phys. 13, 180 (1969). 